\title{
Enhanced Modal Dispersion Estimation Enabled by Chromatic Dispersion Compensation in Optical Vector Network Analysis
}

Van Weerdenburg, John; Rommel, Simon; Mendinueta, Jose Manuel Delgado; Klaus, Werner; Sakaguchi, Jun; Vegas Olmos, Juan Jose; Koonen, Ton; Awaji, Yoshinari; Monroy, Idelfonso Tafur; Okonkwo, Chigo

Total number of authors:

11

Published in:

Journal of Lightwave Technology

Link to article, DOI:

10.1109/JLT.2019.2923112

Publication date:

2019

Document Version

Peer reviewed version

Link back to DTU Orbit

Citation (APA):

Van Weerdenburg, J., Rommel, S., Mendinueta, J. M. D., Klaus, W., Sakaguchi, J., Vegas Olmos, J. J., Koonen, T., Awaji, Y., Monroy, I. T., Okonkwo, C., \& Wada, N. (2019). Enhanced Modal Dispersion Estimation Enabled by Chromatic Dispersion Compensation in Optical Vector Network Analysis. Journal of Lightwave Technology, 37(16), 4001-4007. [8736774]. https://doi.org/10.1109/JLT.2019.2923112

- You may freely distribute the URL identifying the publication in the public portal 


\title{
Enhanced Modal Dispersion Estimation Enabled by Chromatic Dispersion Compensation in Optical Vector Network Analysis
}

\author{
John van Weerdenburg, Student Member, IEEE Simon Rommel, Member, IEEE, Member, OSA, \\ José Manuel Delgado Mendinueta, Member, IEEE Werner Klaus, Member, IEEE Jun Sakaguchi, Member, IEEE \\ Juan José Vegas Olmos, Senior Member, IEEE Ton Koonen, Fellow, IEEE, Yoshinari Awaji, Member, IEEE \\ Idelfonso Tafur Monroy, Senior Member, IEEE, Chigo Okonkwo, Senior Member, IEEE, and
} Naoya Wada, Member, IEEE

\begin{abstract}
Component characterization is fundamental for understanding the limits of optical devices, sub-systems and transmission systems. With the introduction of space division multiplexing in optical fiber transmission systems, new impairments such as mode dependent loss and differential mode dispersion arise. Spatially-diverse optical vector network analyzers are capable of measuring these characteristics in a fast single sweep over a very large bandwidth. As result of this large bandwidth, these analyzers are sensitive to differential chromatic dispersion within the interferometric measurement setup. This work discusses the influence and compensation of differential chromatic dispersion in such systems. Partial chromatic dispersion compensation is demonstrated to improve the representation and accuracy of impulse response measurements obtained from optical vector network analyzers for fibers and components with large differential chromatic dispersion. Analysis of a 39 core few-mode multi-core fiber is discussed, reporting variances of $-2.9 \mathrm{ps} / \mathrm{nm}$ to $-0.1 \mathrm{ps} / \mathrm{nm}$, and $0.6 \mathrm{ps} / \mathrm{nm}$ to $6.9 \mathrm{ps} / \mathrm{nm}$ for the two mode groups, respectively, between the few-mode cores. A correlation with the total impulse response is observed. Furthermore, a maximum propagation skew of $20 \mathrm{~ns}$ between cores is observed after $13.6 \mathrm{~km}$.
\end{abstract}

Index Terms-Space division multiplexing, optical vector network analysis, mode dependent loss, differential mode dispersion, device characterization, component analysis.

Manuscript received December 13, 2018; revised June 03, 2019, accepted MMM DD, YYYY. This work was supported by the Dutch NWO Photonics Graduate programme under GA 022.005 .011 and by the blueSPACE project which has received funding from the European Union's Horizon 2020 research and innovation programme under grant agreement No. 762055.

John van Weerdenburg, Ton Koonen, I. Tafur Monroy and Chigo Okonkwo are with the Institute for Photonic Integration, Eindhoven University of Technology, 5600 MB Eindhoven, The Netherlands (e-mail: j.j.a.v.weerdenburg@tue.nl).

S. Rommel was with the Department of Photonics Engineering, Technical University of Denmark, $2800 \mathrm{Kgs}$. Lyngby, Denmark and with the Photonic Network System Laboratory, National Institute of Information and Communication Technology (NICT), 4-2-1 Nukui-Kitamachi, Koganei, Tokyo 184 8795, Japan. He is now with the Institute for Photonic Integration, Eindhoven University of Technology, 5600 MB Eindhoven, The Netherlands (e-mail: s.rommel@tue.nl).

José Manuel Delgado Mendinueta, Werner Klaus, Jun Sakaguchi, Yoshinari Awaji and Naoya Wada are with the Photonic Network System Laboratory, National Institute of Information and Communication Technology (NICT), 4-2-1 Nukui-Kitamachi, Koganei, Tokyo 184-8795, Japan.

Juan José Vegas Olmos is with Mellanox Technologies, Ledreborg Allé 130B, 4000 Roskilde, Denmark.

Color versions of one or more of the figures in this paper are available online at http://ieeexplore.ieee.org.

Digital Object Identifier 10.1109/JLT.2019.XXXXXX

\section{INTRODUCTION}

$\mathbf{O}$ PTICAL communication technology has advanced rapidly in recent years, supporting our increasingly information-driven society. The main prognosis is that demand will exceed the available capacity in the widely deployed single-mode fiber based systems in the near future [1], [2]. As a consequence, research in space-division multiplexing (SDM) has demonstrated transmission rates beyond $10 \mathrm{Pbit} / \mathrm{s}$ by exploiting multiple spatial channels in a single fiber [3]. Spatial multiplicity can be realized by exciting multiple spatial modes within a single few-mode or multi-mode core [4]-[7], transmitting over multiple cores in a single cladding [8], or by a combination of core and mode multiplexing [3], [9], [10].

The key to unlocking the potential capacity in SDM transmission systems is the characterization of individual components and sub-systems, as well as understanding the impairments introduced by those. One performance limiting effect are differences in losses and gains between the spatial channels, referred to as mode dependent loss (MDL). Components with large MDL can reduce the total system capacity, or, in extreme cases, can lead to channel outage [11]. Compensating this impairment is challenging, thus minimizing MDL must be mainly addressed in component design and manufacturing. Next to the different losses, the spatial channels propagate at different speeds through the system. A large variation between the modal group delays, or differential mode delay (DMD), leads to an increase of the impulse response duration, which in turn increases the memory required for the multipleinput multiple-output (MIMO) digital signal processing (DSP) algorithms employed to compensate mode mixing [12] and causes equalization enhanced phase noise [13].

Measurements for both MDL and differential group delay (DGD) can be quantified by the DSP algorithms in transmission experiments. Besides the large and expensive setup required, the measured bandwidth is typically limited to a single wavelength channel at a time. Hence, measuring the full C- or L-band for a temporally unstable system is challenging. Optical vector network analysis (OVNA) is a proven technique for component characterization over an extensive wavelength range in a single fast sweep measurement [14]-[18]. Such systems, based on swept wavelength interferometry (SWI) 


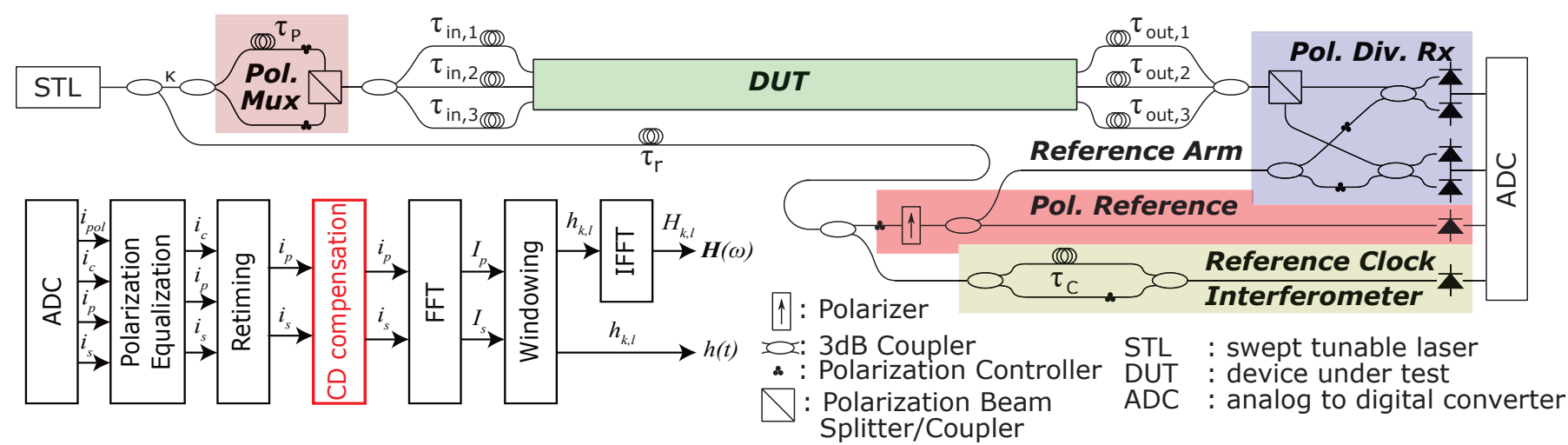

Figure 1: Spatially-diverse optical vector network analyzer setup and digital signal processing steps.

employ a swept laser source that is fed to an interferometric structure, resulting in a fringe pattern dependent on the laser tuning rate and interferometer arm length differences. A device under test (DUT), placed in one of the arms will affect the interference pattern according to its transfer function matrix $\boldsymbol{H}(\omega)$. From this interference pattern, the $2 \times 2$ Jones matrix of a single-mode component can be extracted. Subsequently, linear device parameters such as insertion loss (IL), group delay (GD), chromatic dispersion (CD), polarization dependent loss (PDL) and polarization mode dispersion (PMD) can be obtained from this matrix. The time-windowing technique proposed for measuring multi-port devices [17], can be applied to capture the $2 M \times 2 N$ transfer matrices of SDM components [15], [16], [19]. To measure a $2 M \times 2 N$ transfer matrix, an $\mathrm{M}$-port splitter and an $\mathrm{N}$-port combiner are required. The inherent power losses of the components challenge system scalability to a high number of modes. This especially holds for coupled spatial channels, such as modes in a few-mode fiber (FMF). Requirements for multi-core fiber (MCF) are slightly relaxed, as the crosstalk between the cores is typically designed to be small. Consequently, each core can be measured independently, reducing the splitting dimension and hence losses of the system. In addition to the previously mentioned linear device parameters, their multi-dimensional variants for SDM systems, i.e., MDL and DGD, can be calculated from the transfer function matrix.

In this work, we extend the detailed discussion on optical vector network analyzer (OVNA) for SDM components given in [16], by addressing the measurement procedure of the phase derived properties GD and CD. Both measured quantities include parts introduced by the DUT, and parts originating from the different interferometer lengths introduced to measure multi-port components. A residual contribution related to the mismatch with the reference arm is common to all matrix elements of transfer function matrix $\boldsymbol{H}(\omega)$, and can be estimated. Similar to digital CD compensation in coherent optical transmission, this undesired dispersion can be removed with DSP, improving the accuracy of impulse response duration measurements. CD compensation is particularly beneficial for characterization of longer components, such as fiber. Furthermore, digital $\mathrm{CD}$ compensation is demonstrated to relax the requirement of matching interferometer arm lengths.
Optical vector network analyis of a large core-count fewmode multi-core fiber (FM-MCF) shows a large difference between the cores [20]. A detailed analysis of the impulse response duration and wavelength dependent dispersion is performed, reporting impulse response durations between $0.6 \mathrm{~ns}$ and $3.0 \mathrm{~ns}$. Differential $\mathrm{CD}$ with respect to a single-mode core within the FM-MCF is $-2.9 \mathrm{ps} / \mathrm{nm}$ to $-0.1 \mathrm{ps} / \mathrm{nm}$ for the $\mathrm{LP}_{01}$ and $0.6 \mathrm{ps} / \mathrm{nm}$ to $6.9 \mathrm{ps} / \mathrm{nm}$ for the $\mathrm{LP}_{11}$ mode groups, respectively. Partial compensation is applied on the individual mode groups, to remove the spreading of the impulse response introduced by $\mathrm{CD}$, improving the accuracy of modal dispersion estimates. Furthermore, difference in group delay of the fewmode cores with respect to the single mode core up to $20 \mathrm{~ns}$ are observed.

The remainder of this article is structured as follows: Section II introduces the hardware system and DSP required for characterization of SDM components using a spatially-diverse OVNA mainly focused on the measurement of wavelength dependent dispersion. The digital $\mathrm{CD}$ compensation and its impact on OVNA measurements is also discussed here. In Section III, a 39-core 3-mode FM-MCF is analyzed using OVNA. Finally, Section IV concludes the article.

\section{DESCRIPTION OF A SPATIALLY-DIVERSE OPTICAL VECTOR NETWORK ANALYZER}

A comprehensive description of spatially diverse optical vector network analyzers is given in [16]. The presented analysis is based on the group delay, derived from the frequency dependent propagation constant of the optical field, but ignores any higher order terms. This assumption is accurate when these effects are negligibly small, such as for slow sweeping rates and short device lengths. However, for optical vector network analysis of long fibers, higher order transmission effects encaptured within the propagation constant have to be taken into account.

The swept tunable laser (STL) source of the spatially-diverse OVNA, depicted in Fig. 1 produces an optical field:

$$
\boldsymbol{E}_{S T L}(t)=A_{S T L}(t) \mathrm{e}^{-j \omega(t) t} \boldsymbol{p}_{S T L}
$$

where $A_{S T L}$ is the complex amplitude and $\boldsymbol{p}_{S T L}$ is the polarization state at the output of the STL. The angular frequency $\omega(t)=2 \pi c /\left(\lambda_{s}+\nu(t) t\right)$ is the result of the sweep 
starting at $\lambda_{s}$ with a sweep rate of $\nu(t)$. After propagation through an optical fiber of length $z$, the field is given by:

$$
\boldsymbol{E}(z, t)=A(z, t) \mathrm{e}^{-j \omega t} \mathrm{e}^{-j \beta(\omega) z} \boldsymbol{p}_{z},
$$

where $\beta(\omega)$ is the frequency dependent propagation constant, and $\boldsymbol{p}_{z}$ is the polarization vector at point $z$. Analogue to the derivation in [16], but with the inclusion of the propagation constant $\beta$, the expression for one of the photo-currents from the polarization diverse receiver is given by:

$$
\begin{array}{r}
i_{s}=\mathcal{R}|A|^{2} \frac{\sqrt{\kappa}}{N} \frac{\sqrt{1-\kappa}}{\sqrt{2}} \sum_{k, l=1}^{N} \operatorname{Re}[ \\
\left(s_{1} \hat{H}_{2 k-1,2 l-1}+s_{2} \hat{H}_{2 k, 2 l-1}\right) \mathrm{e}^{-j\left[\beta\left(z_{\text {out }, k}+z_{\text {in }, l}-z_{R}\right)+\omega(t) t\right]} \\
\left.+\left(s_{1} \hat{H}_{2 k-1,2 l}+s_{2} \hat{H}_{2 k, 2 l}\right) \mathrm{e}^{-j\left[\beta\left(z_{\text {out }, k}+z i n, l+z_{P}-z_{R}\right)+\omega(t) t\right]}\right],
\end{array}
$$

where $\kappa$ denotes the asymmetrical beam-splitting ratio at the source, $\mathcal{R}$ denotes the sensitivity of the square law photodetectors, and $z$ denote the fiber lengths in the system. The transfer function elements are denoted as $\hat{H}_{k, l}$, where $k$ and $l$ represent the inputs and outputs of the DUT. The photo-current of the other detector is found by replacing the polarization vector $s$ with the orthogonal vector $\boldsymbol{p}$ of the polarization beam combiner $(\mathrm{PBC})$ in the polarization diverse receiver. Note that all contributions related to the delay lines $\tau_{p}, \tau_{i n}$, and $\tau_{\text {out }}$ can be calibrated out by reference measurements taken for the back-to-back configuration of the system, i.e., directly connecting all possible combinations of input delay lines with every output delay lines. However, the delay associated with the path length difference of DUT and reference arm, denoted by $z_{r}$ changes for each DUT is not included in the this calibration.. The contribution of $z_{r}$ is decomposed by approximating the propagation constant $\beta$ with a Taylor expansion around $\omega_{0}$ :

$$
\beta\left(\omega_{0}\right)=\sum_{n=0}^{\infty} \frac{\beta^{(n)}\left(\omega_{0}\right)}{n !}\left(\omega-\omega_{0}\right)^{n},
$$

where $\beta^{(n)}\left(\omega_{0}\right)$ denotes the $n^{\text {th }}$ derivative of $\beta$ with respect to $\omega$ evaluated at $\omega_{0}$, and $n$ ! is the factorial of $n$. The first element of the sum $(n=0)$, produces a constant phase factor independent of the angular frequency, and thus can be neglected for this application. The contribution of $n=1$ is related to the group delay $\tau_{z}$ associated with path length $z$, which translates to a frequency shift. This property is exploited to detect multiple transfer functions simultaneously in the polarization and space diverse OVNA setup. The group velocity dispersion (GVD) or CD described by $\beta^{(2)}$, introduces pulse broadening as result of different propagation speeds of spectral components. Since it is difficult to distinguish different types of dispersion within impulse response graphs, compensating the differential $\mathrm{CD}$ with respect to the reference arm can improve modal dispersion estimations from these types of graphs. Optionally, higher order effects described by $\beta$ can be taken into account. However, from the performed measurements, higher order propagation effects deemed small enough to be of no relevance for modal dispersion estimation in few-mode fibers covered in this work. Hence, no additional higher order propagation effects are considered.

\section{A. Digital signal processing for optical vector network analysis}

The first step in the DSP chain, which is depicted in Fig. 1, compensates the power fading related to the wavelength dependent polarization changes in the reference arm [21]. A moving average filter based on the polarized reference signal is calculated and subtracted from the two data signals.

The next module, converts the non-linear wavelength sweep to a linear frequency sweep by extracting new sample points from the received signal of the auxiliary clock interferometer. A bandpass-filter is applied around the expected clock frequency before detecting all zero crossings of the signal. The resulting sampling vector is upsampled and applied to both data channels to obtain waveforms linearly spaced in frequency. In the spectra, obtained via FFT, the impulse response elements $h_{k l}$ are found at the positions corresponding to the fiber delay $\tau_{p}, \tau_{i n}, \tau_{\text {out }}$, and $\tau_{\text {ref }}$. Each element is selected by applying a windowing function. A flat top windows with sharp edges is applied to reduce ringing due to edge effects, while preserving the device impulse response in the center. Finally the frequency response elements $H_{k l}(\omega)$ are found by taking the IFFT of $h_{k l}(t)$. Note that in the windowing process, the optical frequency sample spacing is reduced to one over the duration of the window.

From $\boldsymbol{H}(\omega)$, both MDL and IL can be retrieved via singular value decomposition at every frequency [11], [15], [16]. The resulting $\lambda_{i}(\omega)$ are the channel gains of the eigenmodes of the DUT. The IL is calculated as the mean of the $\left|\lambda_{i}(\omega)\right|^{2}$. Taking the ratio between largest and smallest singular value returns the MDL.

Next to the singular value analysis of $\boldsymbol{H}(\omega)$, an eigenvalue analysis on $\boldsymbol{H}(\omega+\delta \omega) \boldsymbol{H}(\omega)^{-1}$ can be performed, from which the group delays $\tau_{g}$ and DMD of the principal modes are obtained [11]. Taking the derivative of these group delays with respect to wavelength leads to the GVD of the principal modes. Alternatively, the DMD can also be obtained directly from the impulse response matrix $\boldsymbol{h}(t)$ by measuring the time delay between mode peaks. This estimation can be performed on the individual matrix elements $h_{i j}(t)$ or in a single estimation on the sum of the squared elements: $\sum\left|h_{i j}\right|^{2}$.

Note that both estimations cover the complete measurable range of the OVNA, and thus for components with strong wavelength dependent behaviour, i.e., devices with large differential $\mathrm{CD}$, the impulse response duration will be overestimated. For this reason, a visual representation covering both time and frequency behaviour in a single graph is preferred. Modes and mode groups are generally easier to recognize in such spectrograms, allowing labeling of the mode groups. From these graphs, not only DMD estimations can be derived, they also visualize the wavelength dependent change of dispersion. One notable disadvantage of these spectrograms is their reduced visual resolution as a result of using a color-scale to represent transmission intensity compared to standard lines in an impulse response plot. Analog to the impulse response, 


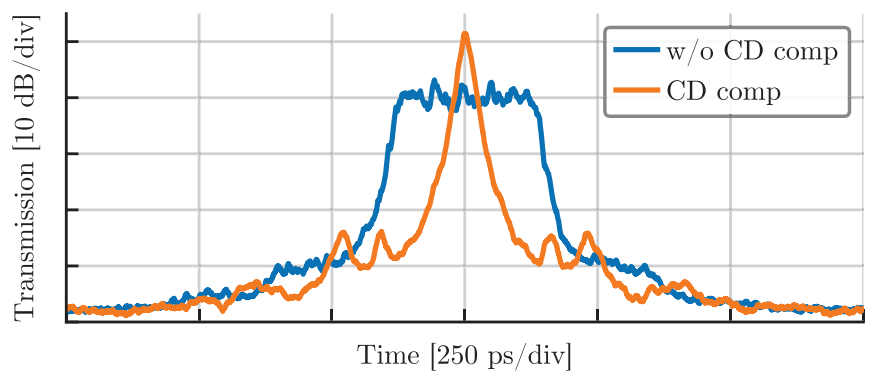

Figure 2: Impulse response of a single-mode fiber core before (a) and after (b) compensating differential chromatic dispersion.

polarization or mode collapsed versions of these graphs can be obtained by summing matrix elements.

\section{B. Digital chromatic dispersion compensation in optical vector network analyzers}

As described at the beginning of this section, the obtained transfer function matrix includes propagation effects related to the differential path length of DUT and reference arm, denoted by $\tau_{r}$. In order to improve modal dispersion estimation from impulse response graphs, additional sources of dispersion must be removed from the calculated transfer function matrix. Hence, digital dispersion compensation, similar to coherent optical communication, is applied [22]. The following digital filter with a complementary amount of dispersion is designed and applied to the signals:

$$
H_{C D}(z, \omega)=\exp \left(-j \frac{D \lambda_{0}^{2}}{4 \pi c} \omega^{2}\right),
$$

where $\lambda_{0}$ is the center wavelength of the sweep, $c$ denotes the speed of light, and $D$ denotes the accumulated CD over a path length $z$.

An example of the effect of $\mathrm{CD}$ compensation on impulse response measurements with OVNA is shown in Fig. 2, which shows the impulse response of a single mode fiber core. From OVNA a differential CD of $16.4 \mathrm{ps} / \mathrm{nm}$ with the reference arm was observed. This value is verified by optical timedomain reflectometry (OTDR) measurements. This differential $\mathrm{CD}$ is mainly attributed to the $46 \mathrm{~m}$ length differences of DUT and standard single-mode fiber (SSMF) span employed as reference. As can be seen in Fig. 2, the impulse response spread is significantly reduced by applying a filter designed with $-16.4 \mathrm{ps} / \mathrm{nm}$ dispersion.

\section{AnAlysis of A 39-CORE FEW-MODE FIBER}

This section covers the analysis of a 39 core FM-MCF using OVNA by exploiting the setup in Fig. 1. A detailed depiction of the DUT section of the setup is shown in Fig. 3a. After polarization multiplexing with a delay $\tau_{\text {pol }}=50 \mathrm{~ns}$, three input delays $\tau_{i n, k}=k \cdot 4.5 \mathrm{~ns}$ for the modes are placed at the input of a laser inscribed glass mode-multiplexer. The few-mode output pigtail is switched between the core multiplexer input ports, which are equipped with specially designed SC/UPC few-mode connectors [23]. At the other side of the $13.6 \mathrm{~km}$

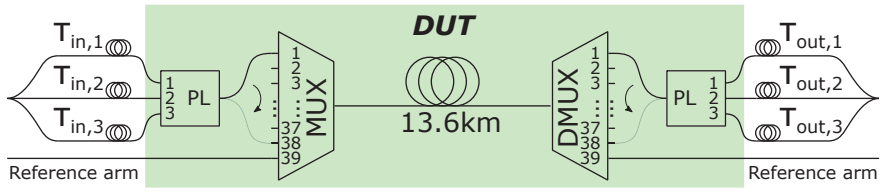

(a)

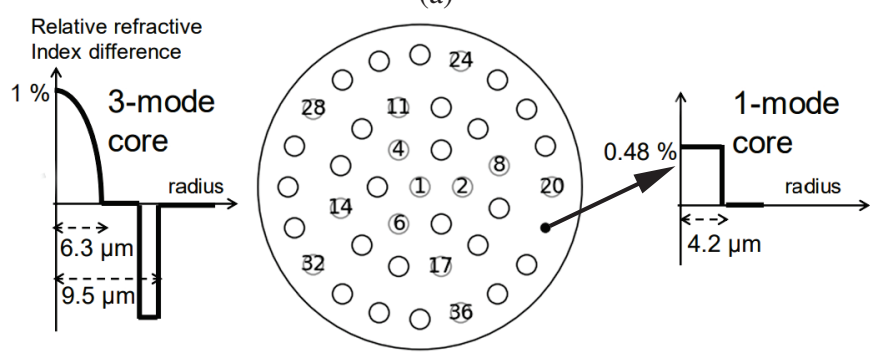

(b)

Figure 3: (a) DUT section placed in setup of Fig. 1. The reference arm is guided through the single mode core, core 39. (b) Core layout of the 39 core fiber and the refractive index profile of the 3-mode and single-mode cores.

fiber, a reciprocal configuration including mode- and coredemultiplexer is configured with delays $\tau_{\text {out }, l}=l \cdot 45 \mathrm{~ns}$. Note that $\tau_{\text {in, } 0}$ and $\tau_{\text {out }, 0}$ are typically omitted in similar setups. However, for this configuration, where the reference arm has exactly the same length as the DUT, additional delay was introduced to avoid the risk of overlapping signals in the spectrogram. The transfer function matrix for each core is obtained in a single sweep, at a rate of $100 \mathrm{~nm} / \mathrm{s}$ ranging from $1530 \mathrm{~nm}$ to $1570 \mathrm{~nm}$. All signals are digitized by a real-time oscilloscope sampling at $10 \mathrm{MSa} / \mathrm{s}$, followed by the DSP steps of the schematic in Fig. 1. The optical sample rate produced by the $20 \mathrm{~m}$ delay in the reference clock interferometer is 16 times digitally up-sampled before re-timing the measurement channels.

\section{A. Large core-count few-mode fiber}

The core layout of the $312 \mu \mathrm{m}$ cladding diameter fiber under test is shown in Fig. 3b. The cores are placed with a minimum pitch of $40 \mu \mathrm{m}$ on a three-ring layout with radii $r_{1}=41 \mu \mathrm{m}$, $r_{2}=79 \mu \mathrm{m}$, and $r_{3}=127 \mu \mathrm{m} .38$ of the cores are of the trench assisted graded-index type, designed to support 3-modes each. The last core supports only the fundamental mode, and thus can be employed in self-coherent detection schemes [24][27], or - as in this work - as a reference arm for OVNA. Furthermore, it serves as a visible reference marker for core alignment.

\section{B. Analysis results and discussion}

The measurement of each core results in an impulse response matrix $\boldsymbol{h}(t)$ and transfer function matrix $\boldsymbol{H}(\omega)$ of dimension 6, which is represented by a single spectrogram for each core in Fig. 4. Note that difference between the individual elements are minimal, because of the employed non mode-selective photonic lanterns (PLs). In each spectrogram, multiple temporal lines can be seen. Although the position and 
core 5

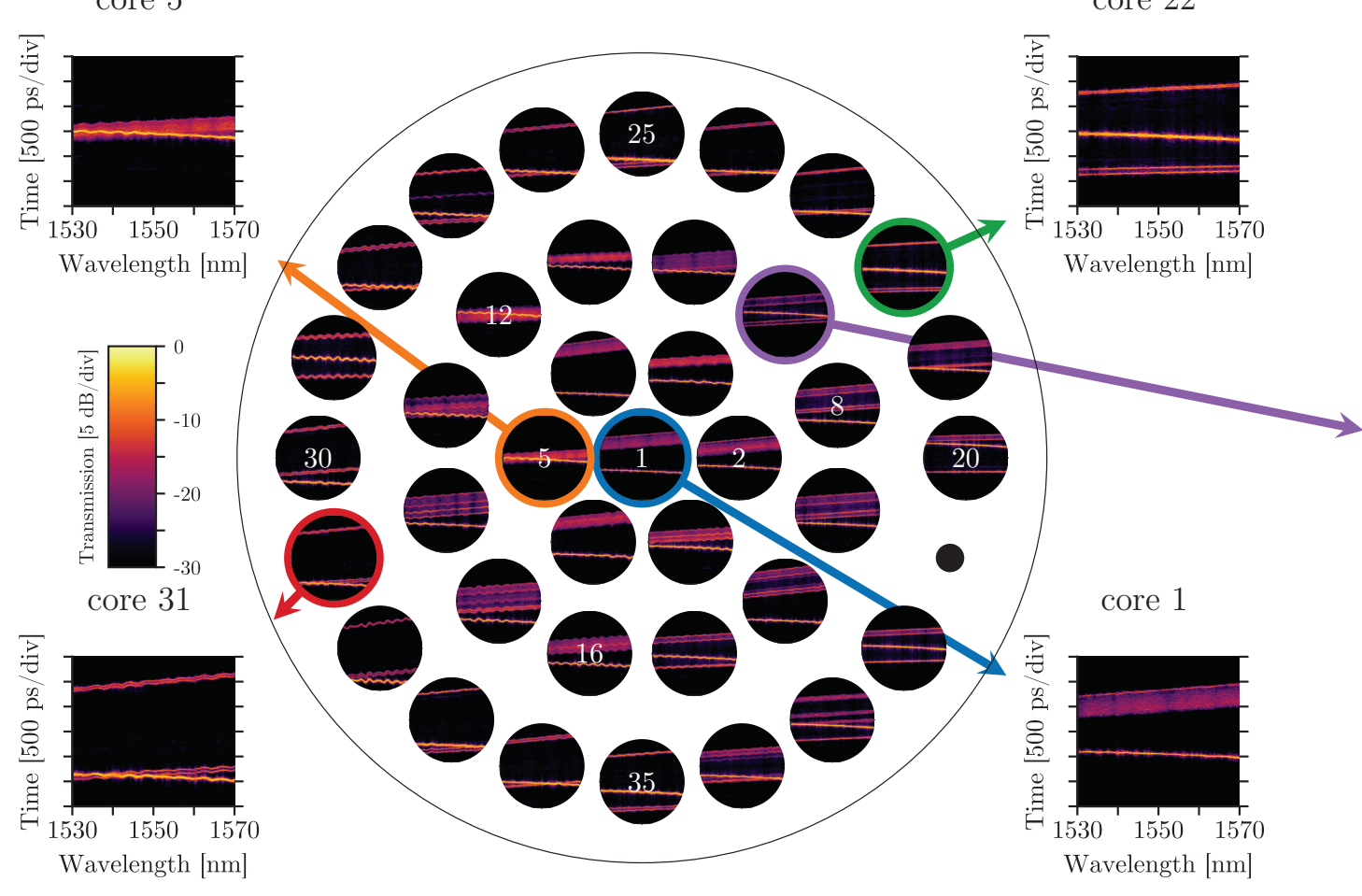

core 9

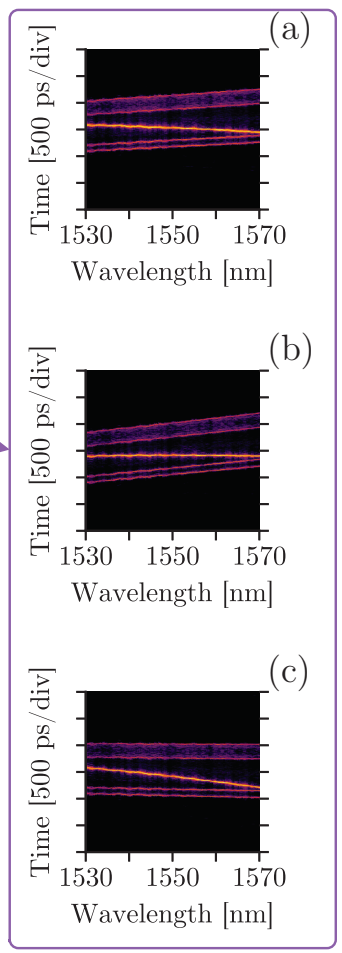

Figure 4: Spectrograms for all 38 few-mode cores, with enlarged images for cores 1, 5, 22 and 31 . Differential CD is demonstrated for core 9. (a) spectrogram without compensation, (b) $-2.3 \mathrm{ps} / \mathrm{nm}$ compensation for $\mathrm{LP}_{01} \mathrm{mode}$, and (c) $2.8 \mathrm{ps} / \mathrm{nm}$ compensation for $\mathrm{LP}_{11}$ modes.

slopes differ strongly between the cores, they can be grouped in two sets based on the direction of the slope. For every core, two closely confined lines with a negative slope are observed. Due to the small differential group delay between them, these temporal lines are linked to the two polarization states of the fundamental mode. The other set contains four lines, which correspond to the degenerate modes of the $\mathrm{LP}_{11}$ group. Note that the differential group delay varies strongly between the cores. The spectrograms of cores 1, 5, 22 and 31 are selected as examples to discuss recognizable features. Therefore, enlarged views of these spectrograms are included in Fig. 4.

In the spectrogram of core 5 , both groups slightly diverge but overlap for the $40 \mathrm{~nm}$ of analyzed wavelength range, indicating the mode coupling between the groups is stronger and DMD is smaller compared to the other depicted cores in Fig. 4. The spectrogram of core 22 shows a clear separation between the mode groups. Based on the dispersion slope of the lines it is presumed that the center line represents the $\mathrm{LP}_{01}$ mode, which is surrounded by the degenerate $\mathrm{LP}_{11}$ modes. The wavelength dependent impulse response spread for this core, in this $40 \mathrm{~nm}$ range is completely determined by the $\mathrm{LP}_{11}$ mode. Core 31 also shows a noticeable amount of differential mode group delay (DMGD), but there is a clear overlap between the fundamental mode and one of the $\mathrm{LP}_{11}$ modes. The last type of spectrogram is illustrated by core 1 , which shows a clean separation between the two mode groups with a significant modal dispersion of the four degenerate $\mathrm{LP}_{11}$ modes.
From the spectrogram arrangement to the core layout in Fig. 3b, it is observed that the cores on the left half of the outer ring closely resemble the behavior depicted in the spectrogram for core 31 and the right half of that circle behaves similar to core 22. A similarly low DMD characteristic as in core 5 (Fig. 4a) is also observed for core 12. Similar to core 1, a larger impulse response spread for the $\mathrm{LP}_{11}$ modes for cores on the inner circles is observed. The strongly varying behavior of the few-mode cores is likely related to imperfections of

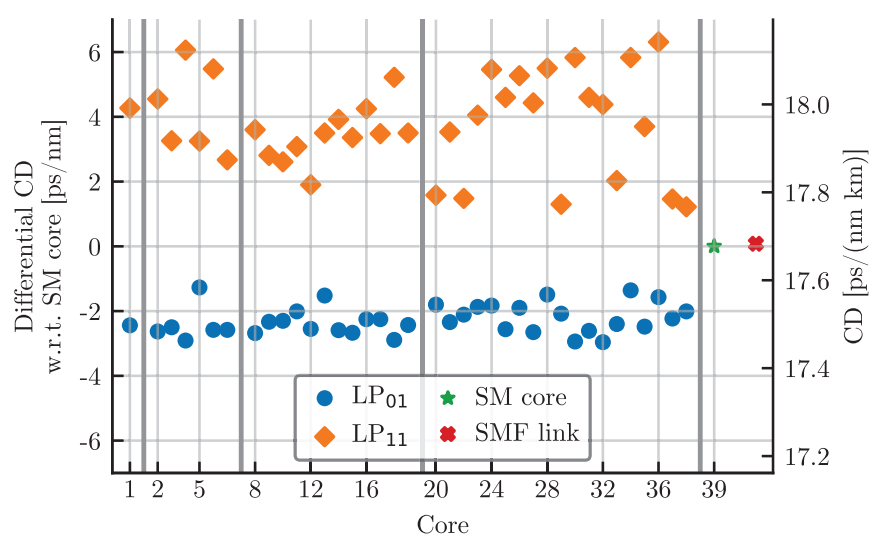

Figure 5: (Differential) chromatic dispersion for all modes and cores. The values of the single-mode core, and single-mode fiber (SMF) link are included for reference. 


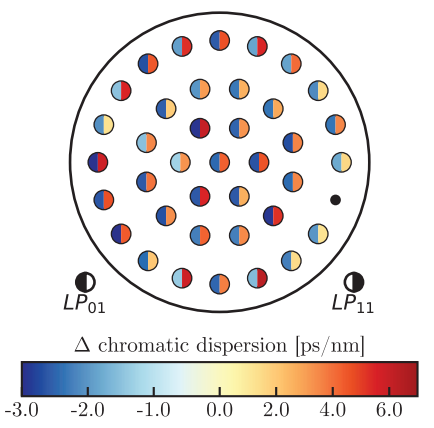

(a)

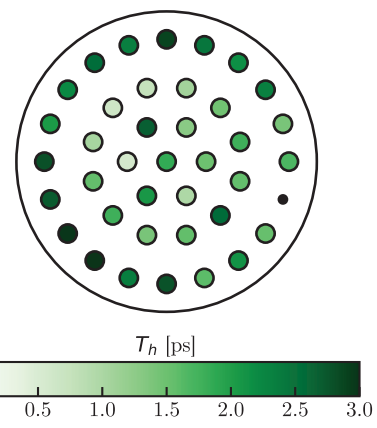

(b)

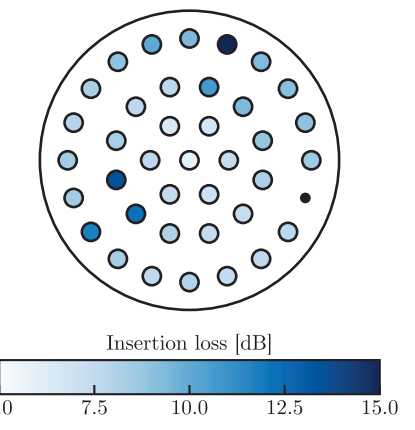

(c)

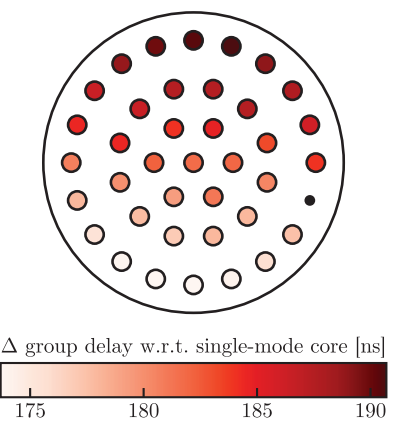

(d)

Figure 6: (a) Differential CD of of all cores mapped to the core-layout. (b) Total impulse response duration for all cores. (c) Insertion losses of the few-mode cores including core- and mode-multiplexers. (d) Group delay difference between few-mode and single-mode core.

the manufacturing process, resulting in deviations from the intended design.

Furthermore, observe the slope of the lines from all spectrograms. For all cores, the two mode groups have a wavelength dependent slope in opposite directions. The slope for both lines was estimated and the resulting $\mathrm{CD}$ for each core is given in Fig. 5. The left axis shows the differential $\mathrm{CD}$ with respect to the single-mode core, and the actual CD in $\mathrm{ps} /(\mathrm{nm} \cdot \mathrm{km})$ is given on the right. This last value was obtained by measuring the $\mathrm{CD}$ of the single-mode core using a fiber analyzer based on OTDR, the SMF span used as a reference to characterize the single-mode core with the OVNA is also included in the graph. The graph shows that in all fiber cores, the fundamental mode has slightly less CD with respect to SMF, whereas the other mode-group has a larger $\mathrm{CD}$ value with a larger spread among the cores.

The measured $\mathrm{CD}$ values are mapped to the core-layout in Fig. 6a, where each half of the circle represents one of the mode groups. This measurement is compared to the previously analyzed impulse response duration in [28], shown in Fig. 6b. A strong correlation between the color intensities of the graph can be observed, linking the longer impulse response duration to a larger differential $\mathrm{CD}$ value. The longest impulse responses and highest CD values are measured for the cores on the left half of the outer ring (core 25-35).

$\mathrm{CD}$ compensation based on the measured values has been

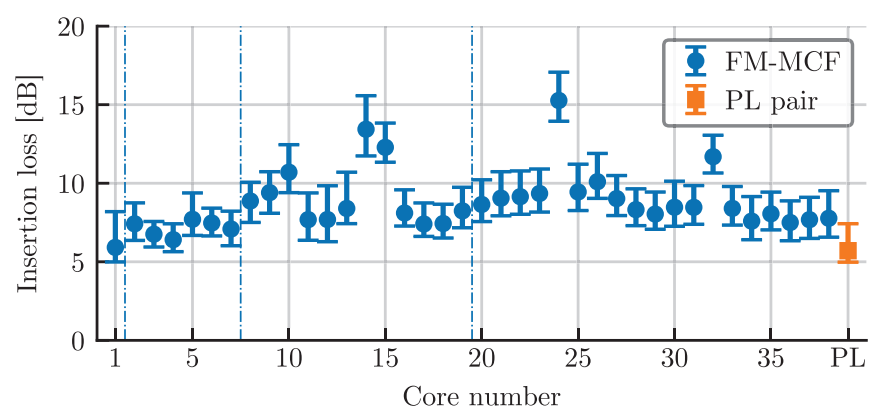

Figure 7: Insertion losses per $50 \mathrm{GHz}$ for each cores, and the pair of lanterns. applied on the cores to isolate the DMGD. Given the different amounts of dispersion for the individual mode groups, only one of the groups can be fully compensated. As can be observed in the spectrograms for core 9 in Fig. 4, where $\mathrm{CD}$ compensation is applied to each individual mode group by applying $-2.3 \mathrm{ps} / \mathrm{nm}$ (Fig. 4b) and $2.8 \mathrm{ps} / \mathrm{nm}$ (Fig. 4c) CD compensation receptively. Alternatively, an intermediate value can be chosen for partial compensation of both mode groups. By removing the wavelength dependent dispersion, the summed impulse responses are narrower, and give a better indication of the modal dispersion.

For each core, a singular value decomposition (SVD) is performed on the transfer function matrix at each frequency. Subsequently, the IL is calculated at $50 \mathrm{GHz}$ resolution. Figure 7 shows the average, minimum, and maximum IL in the band ranging from $1530 \mathrm{~nm}$ to $1570 \mathrm{~nm}$. An average IL of $8.7 \mathrm{~dB}$ is observed between all the few-mode cores, with high insertion losses for cores 10,14, 15, 25 and 32. The obtained IL is in agreement with the system IL measured for the transmission experiment in [23]. Note that the losses includes contributions of the PL mode-multiplexers. Therefore, the IL of the PL pair is measured with the OVNA, from which an average IL of $2.8 \mathrm{~dB}$ per PL is obtained. Furthermore, a similar variation of losses within the $40 \mathrm{~nm}$ range is observed.

For the evaluated DUT, the largest contribution of MDL is to be expected from the spatial multiplexers. Hence, the MDL of the 3-mode multiplexer pair is measured first. An average MDL of $20.1 \mathrm{~dB}$ for $50 \mathrm{GHz}$ sub-bands within the $40 \mathrm{~nm}$ analyzed bandwidth was found. The measured system MDL of both mode- and core-multiplexers, and $13.6 \mathrm{~km} \mathrm{FM-}$ MCF was measured to be all below $25 \mathrm{~dB}$. Since this result is dominated by the large contribution of the spatial multiplexers and the compound MDL of multiple concatenated elements in the weak coupling regime strongly depends on the relative alignment of their respective principle modes, no statement on the MDL of the fiber can be made.

An important parameter for employing the FM-MCF with single-mode core for self-coherent detection is the skew between few-mode cores and single-mode core. Figure 6d, shows the group delay difference of each few-mode core with respect to the single-mode core. A skew ranging from $173.3 \mathrm{~ns}$ to 
$190.7 \mathrm{~ns}$, with an average of $182.2 \mathrm{~ns}$ is observed. Note that the skew is clearly the smallest on one side of the fiber, and largest on the opposite side. This gradient is likely attributed to the spooling on the fiber, which result in compression of the inner cores and stretching of the outer cores.

\section{CONCLUSIONS}

In this work, we have discussed the influence of differential chromatic dispersion in optical vector network analyzers. Chromatic dispersion compensation is demonstrated for a single-mode core and partially for few-mode cores in a multicore fiber.

Optical vector network analysis is employed for the characterization of a 39 core few-mode fiber, showing large variations in temporal response between the cores. Which demonstrates the challenging fabrication of long, homogeneous large-core count few-mode fibers. Differential chromatic dispersion variations between the cores of $-2.9 \mathrm{ps} / \mathrm{nm}$ to $0.1 \mathrm{ps} / \mathrm{nm}$, and $0.6 \mathrm{ps} / \mathrm{nm}$ to $6.9 \mathrm{ps} / \mathrm{nm}$ for the two mode groups respectively were observed. These variances show strong correlations with the measured impulse response durations of $0.6 \mathrm{~ns}$ to $3.0 \mathrm{~ns}$. Furthermore, an approximate $20 \mathrm{~ns}$ variation in differential group delay of the few-mode cores and single-mode core was observed. Its distrubution suggestes this is mainly caused by the spooling of the fiber, which compresses the inner cores.

It is expected that through improved techniques for accurate optical vector network analysis of components, fibers and subsystems for space division multiplexed transmission, improved development of devices and fibers will pave the way towards actual deployment of SDM in the field.

\section{REFERENCES}

[1] W. Klaus et al., "Advanced space division multiplexing technologies for optical networks," J. Opt. Commun. and Netw., vol. 9, no. 4, pp. C1-C11, Apr. 2017.

[2] P. J. Winzer and D. T. Neilson, "From scaling disparities to integrated parallelism: A decathlon for a decade," Journal of Lightwave Technology, vol. 35, no. 5, pp. 1099-1115, March 2017.

[3] D. Soma et al., "10.16 peta-bit/s dense sdm/wdm transmission over low-dmd 6-mode 19-core fibre across c+l band," in 2017 European Conference on Optical Communication (ECOC), Sept 2017, pp. 1-3.

[4] J. J. A. van Weerdenburg et al., "Mode-multiplexed 16-qam transmission over 2400-km large-effective-area depressed-cladding 3-mode fiber," in 2018 Optical Fiber Communications Conference and Exposition (OFC), March 2018, pp. 1-3.

[5] R. Ryf et al., "Mode-multiplexed transmission over 36 spatial modes of a graded-index multimode fiber," in 2018 European Conference on Optical Communication (ECOC), Sept 2018, pp. 1-3.

[6] J. J. A. van Weerdenburg et al., "138-tb/s mode- and wavelengthmultiplexed transmission over six-mode graded-index fiber," Journal of Lightwave Technology, vol. 36, no. 6, pp. 1369-1374, March 2018.

[7] G. Rademacher et al., " $93.34 \mathrm{tbit} / \mathrm{s} /$ mode (280 tbit/s) transmission in a 3-mode graded-index few-mode fiber," in 2018 Optical Fiber Communications Conference and Exposition (OFC), March 2018, pp. $1-3$.

[8] B. J. Puttnam et al., " $2.15 \mathrm{~Pb} / \mathrm{s}$ transmission using a 22 core homogeneous single-mode multi-core fiber and wideband optical comb," in European Conference on Optical Communication (ECOC). Valencia, Spain: IEEE, Sep. 2015, paper PD3.1.

[9] J. Sakaguchi et al., "228-spatial-channel bi-directional data communication system enabled by 39-core 3-mode fiber," in 2018 European Conference on Optical Communication (ECOC), Sept 2018, pp. 1-3.

[10] K. Shibahara et al., "Iterative unreplicated parallel interference canceler for mdl-tolerant dense sdm (12-core x 3-mode) transmission over 3000 km," in 2018 European Conference on Optical Communication (ECOC), Sept 2018, pp. 1-3.
[11] K.-P. Ho and J. M. Kahn, "Linear propagation effects in mode-division multiplexing systems," J. Lightwave Technol., vol. 32, no. 4, pp. 614628, Feb 2014.

[12] S. O. Arkk, K. Ho, and J. M. Kahn, "Group delay management and multiinput multioutput signal processing in mode-division multiplexing systems," Journal of Lightwave Technology, vol. 34, no. 11, pp. 28672880, June 2016

[13] J. M. Delgado Mendinueta et al., "Numerical investigation of the equalization enhanced phase noise penalty for m-quadrature amplitude modulation formats in short-haul few-mode fiber transmission systems with time-domain equalization," Applied Sciences, vol. 8, no. 11, 2018.

[14] B. L. Heffner, "Accurate, automated measurement of differential group delay dispersion and principal state variation using jones matrix eigenanalysis," IEEE Photonics Technology Letters, vol. 5, no. 7, pp. 814-817, July 1993.

[15] N. K. Fontaine et al., "Characterization of space-division multiplexing systems using a swept-wavelength interferometer," in Optical Fiber Communication Conference/National Fiber Optic Engineers Conference 2013. Optical Society of America, 2013, p. OW1K.2.

[16] S. Rommel et al., "Few-mode fiber, splice and sdm component characterization by spatially-diverse optical vector network analysis," Opt. Express, vol. 25, no. 19, pp. 22 347-22 361, Sep 2017.

[17] G. D. VanWiggeren and D. M. Baney, "Swept-wavelength interferometric analysis of multiport components," IEEE Photonics Technology Letters, vol. 15, no. 9, pp. 1267-1269, Sept 2003.

[18] D. K. Gifford, B. J. Soller, M. S. Wolfe, and M. E. Froggatt, "Optical vector network analyzer for single-scan measurements of loss, group delay, and polarization mode dispersion," Appl. Opt., vol. 44, no. 34, pp. 7282-7286, Dec. 2005.

[19] J. Bohn, J. Carpenter, S. Gross, M. Withford, and J. Schröder, "Characterization of laser inscribed on-chip photonic lanterns with different core distances," in 2015 European Conference on Optical Communication (ECOC), Sept 2015, pp. 1-3.

[20] J. J. A. van Weerdenburg et al., "Chromatic dispersion analysis and compensation in a large core-count few-mode multi-core fiber based on optical vector network analysis," in 2019 Optical Fiber Communications Conference and Exposition (OFC), March 2019, pp. 1-3.

[21] — "Polarization equalization in optical vector network analysis for fiber characterization," submitted.

[22] S. J. Savory, "Digital filters for coherent optical receivers," Opt. Express, vol. 16, no. 2, pp. 804-817, Jan 2008.

[23] J. Sakaguchi et al., "228-Spatial-Channel Bi-Directional Data Communication System Enabled by 39-Core 3-Mode Fiber,' Journal of Lightwave Technology, vol. 37, no. 8, pp. 1756-1763, apr 2019. [Online]. Available: https://ieeexplore.ieee.org/document/8625527/

[24] A. Mecozzi, C. Antonelli, and M. Shtaif, "Kramers-kronig coherent receiver," Optica, vol. 3, no. 11, pp. 1220-1227, Nov 2016.

[25] J. Sakaguchi, W. Klaus, B. J. Puttnam, J. M. D. Mendinueta, Y. Awaji, and N. Wada, "Spectrally-efficient seed-lightwave-distribution system using space-division-multiplexed distribution channel for multi-core 3mode-multiplexed dp-64qam transmission," in 2017 European Conference on Optical Communication (ECOC), Sept 2017, pp. 1-3.

[26] S. van der Heide et al., "Single carrier 1 tbit/s mode-multiplexed transmission over graded-index $50 \mu \mathrm{m}$ core multi-mode fiber employing kramers-kronig receivers," in 2018 European Conference on Optical Communication (ECOC), Sept 2018, pp. 1-3.

[27] X. Chen, S. Chandrasekhar, and P. Winzer, "Self-coherent systems for short reach transmission," in 2018 European Conference on Optical Communication (ECOC), Sept 2018, pp. 1-3.

[28] S. Rommel et al., "Measurement of modal dispersion and group delay in a large core count few-mode multi-core fiber," in 2018 European Conference on Optical Communication (ECOC), Sept 2018, pp. 1-3. 